\title{
Whole Tooth Regenerative Therapy Using a Bioengineered Tooth Germ
}

\author{
Kei Nakajima $\cdot$ Masamitsu Oshima $\cdot$ Takashi Tsuji
}

Published online: 21 December 2013

(C) Springer International Publishing AG 2013

\begin{abstract}
Dental disorders, including dental caries and periodontal disease, can cause fundamental problems for oral functions, such as enunciation, mastication and occlusion, as well as general health issues. Tooth regenerative therapies for tissue repair and whole tooth replacement are currently being developed as novel treatment approaches. As a form of bioengineered organ replacement, whole tooth replacement therapy is considered an important model system for nextgeneration regenerative therapy. We recently reported bioengineered tooth replacements after transplantation of a bioengineered tooth germ or mature tooth unit comprising the bioengineered tooth and periodontal tissues. Whole tooth regenerative therapy has the potential to fully restore tooth function, including masticatory potential in response to mechanical stress and perceptive potential for noxious stimulation. In this review, we describe recent findings and technologies underpinning tooth regenerative therapy.
\end{abstract}

\author{
K. Nakajima $\cdot$ T. Tsuji \\ Department of Biological Science and Technology, Graduate School \\ of Industrial Science and Technology, Tokyo University of Science, \\ Noda, Chiba 278-8510, Japan \\ K. Nakajima \\ e-mail: nakajimakei@tdc.ac.jp \\ K. Nakajima \\ Department of Clinical Pathophysiology, \\ Tokyo Dental College, Chiba-shi, Chiba 261-8502, Japan \\ M. Oshima $\cdot$ T. Tsuji \\ Research Institute for Science and Technology, \\ Tokyo University of Science, Noda, Chiba 278-8510, Japan \\ M. Oshima \\ e-mail: m-oshima@rs.noda.tus.ac.jp \\ T. Tsuji $(\bowtie)$ \\ Organ Technologies Inc., Tokyo 101-0048, Japan \\ e-mail: t-tsuji@rs.noda.tus.ac.jp \\ T. Tsuji \\ e-mail: t-tsuji@nifty.com
}

Keywords Organ germ method · Bioengineered tooth · Bioengineered tooth germ $\cdot$ Bioengineered tooth unit . Tooth development $\cdot$ Epithelial-mesenchymal interaction . Cell compartmentalisation - Tooth regeneration · Tooth physiological function · Occlusion · Organ replacement regenerative therapy

\section{Introduction}

Functions of teeth such as enunciation, mastication and occlusion are important components of a healthy life [1]. The tooth is of ectodermal origin, its development is regulated by reciprocal epithelial-mesenchymal interactions [2, 3], and it has distinctive hard tissues that include enamel, dentin, and cementum. Teeth also contain a soft connective tissue such as pulp and are surrounded by another soft connective tissue, periodontal ligament. Both of these tissues contain nerve fibres and blood vessels to maintain tooth homeostasis [4]. Dental disorders, including dental caries, periodontal disease or trauma, cause fundamental problems for oral function and are associated with oral and general health issues [1]. Conventionally, tooth restoration involves replacement with artificial materials including dentures or dental implants $[5,6]$. Although these artificial therapies are widely applied to the rehabilitation for tooth loss, there have been recent improvements that enhance the biological functions underlying tooth movement through bone remodelling [7].

Current advances in future regenerative therapies have been influenced by many previous studies of embryonic development, stem cell biology and tissue engineering technologies $[8,9]$. To restore partial loss of organ function and to repair damaged tissues, attractive regenerative therapy concepts include stem cell transplantation [10] and cytokine therapy targeted to structural and functional diseases [11]. Toothderived stem cells and the cytokine networks that regulate tooth development have been well characterised at the molecular level [7, 12]. Dental pulp stem cells (DPSCs) and stem 
cells from human exfoliated deciduous teeth (SHED) have been isolated from the dental pulp of human permanent third molars and exfoliated deciduous teeth, respectively $[13,14]$. DPSCs and SHED are thought to be tooth tissue-derived stem cells with high proliferative capacities and sufficient potency to develop into odontoblasts, adipocytes and neural-like cells. Apical papilla contain unique stem cells, known as stem cells from apical papilla (SCAP), that have a high proliferative potential, which is reflected by high levels of telomerase activity and multi-potent differentiation capability that produces odontoblasts and adipocytes [15]. Furthermore, periodontal ligament-derived stem cells (PDLSCs), which have also been identified in adult human periodontal ligaments, can be cultured as stem cells in vitro, and can differentiate into all periodontal cell types after transplantation in vivo [16]. These advances can be applied to the repair of the dentin-pulp complex and periodontal tissues, including the alveolar bone, by replacing enriched and purified stem cells [17-19].

The ultimate goal of regenerative therapy is to develop fully functional bioengineered tissues that can replace lost or damaged organs following disease, injury or aging $[9,20]$. In the dental field, tooth regenerative therapy involves the replacement of a lost or damaged tooth with a bioengineered tooth reconstructed from stem cells and with the potential to generate a functional tooth unit, including the whole tooth and periodontal tissue surrounding the alveolar bone [19]. The establishment of tooth replacement regenerative therapy is anticipated in the near future as a novel and successful biological treatment that will provide essential functional recovery of lost teeth to satisfy aesthetic and physiological requirements [21]. Many approaches to replace missing teeth have been evaluated over the past three decades, including three-dimensionally bioengineered teeth and tooth germ generation using biodegradable materials and cell aggregation methods $[19,20,22]$. The first reports of fully functioning bioengineered tooth replacement with correct tissue structure, masticatory ability, responsiveness to mechanical stress and perceptive potential following transplantation into tooth loss region were recently published $[23,24 \bullet, 25 \bullet \bullet, 26 \bullet]$. In this review, we describe the most recent findings and technologies for whole tooth replacement, which have the potential to provide functional recovery and entirely replace the artificial materials currently used in dental replacements.

\section{Tooth Development}

Organs such as hair, glands, kidneys and teeth arise from their respective germs, which are induced by reciprocal interactions between epithelial and mesenchymal tissues in the developing embryo $[2,17]$. The mechanisms of tooth organogenesis are also regulated by reciprocal epithelial and mesenchymal interactions, particularly those involved in stem cell, signalling molecule and transcription factor pathways. In tooth germ development, the dental lamina first thickens (lamina stage), followed by epithelial thickening (placode stage) at the sites of future teeth and subsequent epithelial budding to the neural crest-derived ecto-mesenchyme. Tooth germ formation is initiated at embryonic days (ED) 10-11 in mice by epithelial signals, e.g., FGF8, BMP4, Shh, TNF and Wnt10b. These signals induce the expression of transcription factors, e.g., Barx1, Dlx1/2, Lhx6, Lhx7 Msx1, Pax9 and Gli, in the dental mesenchyme, which then condense around the developing epithelial bud (bud stage) [2, 3, 17]. At ED14 (cap stage), a first enamel knot known as the transient epithelial signalling centre, which expresses several signalling molecules including Shh, BMPs, FGFs and Wnts, is thought to regulate the epithelial-mesenchymal interactions. The terminal differentiation of dental epithelial and dental mesenchymal cells into ameloblasts and odontoblasts, respectively, occurs at ED1618 (bell stage). These progenitor cells secrete a collagenous extracellular matrix that mineralises into the enamel and dentin matrix at the epithelium-mesenchyme interface. Tooth morphogenesis, including tooth size and shape, are thought to be regulated by signalling molecules such as the BMPs and FGF4 emanating from the secondary enamel knots, which regulate the cusp pattern of the mature natural tooth in the early bell stage to form the tooth crown $[17,27]$. The outer mesenchymal cells of tooth germ form the dental follicle, which can generate periodontal tissue, including cementum, periodontal ligament and alveolar bone. After tooth crown formation, tooth root formation is initiated, followed by tooth eruption in the oral cavity.

\section{Technological Developments for Whole Tooth Regeneration}

The current approach to generating ectodermal organs such as teeth, hair follicles and salivary glands is to recreate organogenesis by mimicking the epithelial-mesenchymal interactions that occur in the developing embryo, thereby developing fully functional bioengineered organs from bioengineered organ germ generated from immature stem cells via threedimensional cell manipulation in vitro [20, 21, 28-30]. For tooth regeneration, a concept has been proposed in which a bioengineered tooth germ is transplanted into the recipient jawbone and subsequently develops into a functional mature tooth (Fig. 1A, upper panel) [25••]. Transplantation of a bioengineered mature organ would enable full function immediately in vivo $\left[3,8,26{ }^{\circ}\right]$. Thus, it is also anticipated that it will be possible to transplant a bioengineered tooth unit including a mature tooth, periodontal ligament and alveolar bone to achieve engraftment through physiological bone integration with the recipient's alveolar bone (Fig. 1A, lower panel) [26•]. To achieve whole tooth replacement, the first 
A

3-D manipulation of single cells

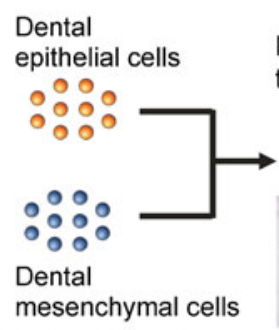

Nakao K. et al.

Nat Methods, 2007 tooth germ
Regeneration of a functional tooth

Eruption of a bioengineered tooth

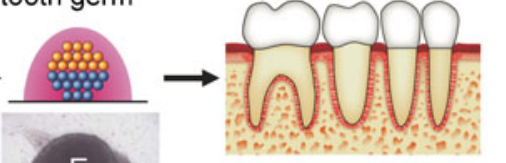

Ikeda E. et al. PNAS, 2009

Replacement of a functional tooth Engraftment of a

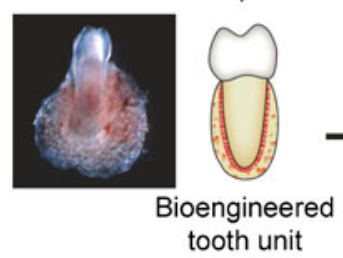
tooth unit

bioengineered tooth unit
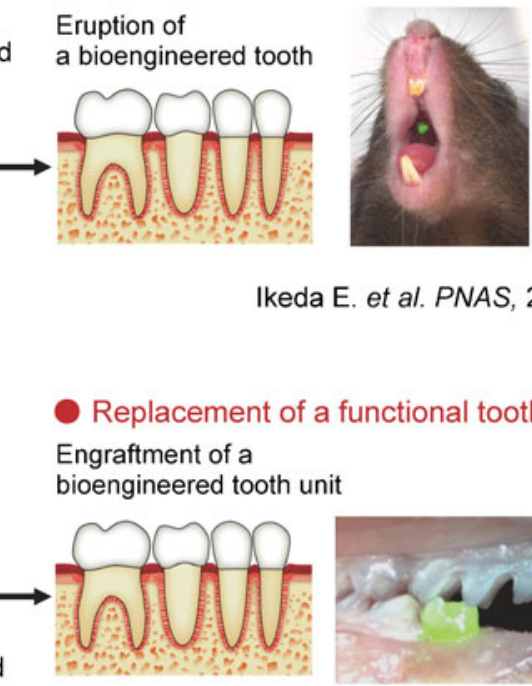

Oshima $\mathrm{M}$

Figure 1 (A) Strategies for the whole tooth replacement regenerative
therapies. Functioning teeth can be regenerated in vivo by transplanting bioengineered tooth germ reconstituted from epithelial and mesenchymal cells via the organ germ method or by transplanting bioengineered tooth units with periodontal ligament and alveolar bone developed from bioengineered tooth germ. E: epithelium, M: mesenchyme. (B) Coordination with the oral and maxillofacial regions. Functional teeth should achieve sufficient masticatory performance with occlusion to the

major issue is to develop a three-dimensional cell manipulation technology using completely dissociated epithelial and mesenchymal cells in vitro. Two conventional approaches and a novel cell manipulation method are currently being examined for the purpose of generating bioengineered tooth germ or mature tooth and are described below.

1) Bioengineering of tooth germ using a biodegradable scaffold

Three-dimensional tissue engineering methods have provided the scaffold technology needed to regenerate suitably shaped tissues by seeding potential cells onto degradable materials fabricated from either natural ingredients or synthetic polymers. This technique has shown high utility in threedimensional tissue engineering methods, and these preparations have been used in clinical applications such as bone and cartilage regenerative therapies $[9,31,32]$. Studies using polyglycolic acid and poly-L-lactate-co-glycolide copolymer (PLA/PLGA) [33-35] or collagen/gelatine sponge as scaffolding materials have reported that seeding epithelial and mesenchymal cells isolated from murine or porcine tooth bud onto a tooth-shaped scaffold could generate small tooth structures, including pulp, dentin and enamel, but not periodontal tissue $[19,21,35-39]$. Although the scaffold method would be
B

Recovery of physiological function

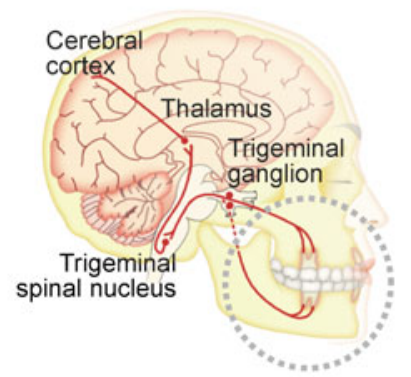

Proper responsiveness via sensory receptors to noxious stimulations

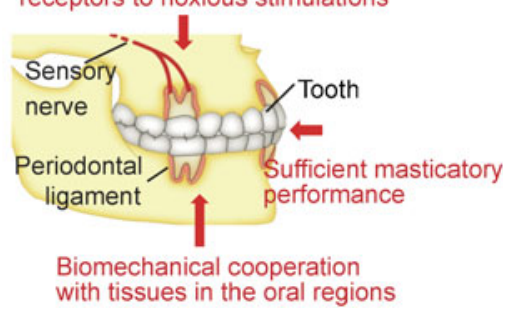

opposing teeth. Tooth function is also responsive to mechanical stress and noxious stimulations, in which it cooperates with both the oral and maxillofacial regions. Noxious stimulations such as mechanical stress and pain, which are detected by the sensory nerves in the pulp and by the periodontal ligament of the tooth, are transduced by the corresponding perceptions of the central nervous system via the trigeminal spinal nucleus in the medullary dorsal horn

practical for tooth shape and size control, it has critical limitations, including tooth formation frequency and the appropriate replication of tooth tissue structures such as the enamel-dentin complex formation that underlies proper arrangements of ameloblast and odontoblast cell lineages, which are achieved through epithelial-mesenchymal interactions as well as natural tooth development $[4,21]$.

2) Reconstruction of tooth germ using cell aggregation methods

Cell aggregation is a typical bioengineering protocol that aims to reconstitute a bioengineered organ germ that properly reproduces epithelial-mesenchymal interactions and organogenesis $[21,38]$. Transplantation of bioengineered cell aggregates using hair follicles and mammary gland-derived stem cells has shown promise for regenerating correct organ structures with proper arrangements of different cell types in vivo $[30,40]$. Tooth germs constructed from precipitates of dental epithelial and mesenchymal cells by using cellular centrifugation can result in complete tooth formation [41, 42]. Mixed cell aggregates of dissociated epithelial and mesenchymal cells isolated from molar tooth germ can also develop into a tooth with the correct structure, following epithelial cell sorting and subsequent epithelial and mesenchymal cell selfreorganisation [43]. Although this technique reconstructs 
organogenesis, the frequency of tooth development and tissue formation is insufficient.

3) Novel three-dimensional cell manipulation methods for bioengineered tooth germ: the "Organ Germ Method"

To achieve precise replication of the processes associated with organogenesis, an in vitro three-dimensional novel cell manipulation method designated as a bioengineered organ germ method has been developed [23, 24•]. This method involves cell compartmentalisation of epithelial and mesenchymal cells from mouse embryonic cap-stage tooth germs at high cell density in a type I collagen gel to mimic multicellular assembly and epithelial-mesenchymal interactions as well as natural tooth development. The bioengineered tooth germ generates a structurally correct tooth after transplantation in an organ culture in vitro, as well as following placement into a subrenal capsule in vivo [23]. Tooth morphology is defined by the macromorphological features of crown size and tooth length, and the micromorphological features of the number and position of the cusps. The crown size is based on the reaction-diffusion model, and the regulation of the cusp number and position is thought to be closely involved in the formation of the secondary enamel knot [17]. Bioengineered tooth germs were reconstructed using various contact lengths between the epithelial and mesenchymal cell layers, and thereby the crown widths and cusp numbers of these bioengineered teeth were dependent on the contact length of the bioengineered tooth germs [44•]. These technologies have the potential to be adapted for successful functional tooth replacement in vivo, and are expected to represent an innovative advance in bioengineered organ replacement regenerative therapies.

\section{Functional Tooth Replacement Regenerative Therapy}

Teeth have important oral functions such as mastication, pronunciation, and facial aesthetics, which have an important influence on quality of life [1]. These tooth-related functions are achieved along with masticatory muscles and the temporomandibular joint under the control of the central nervous system (Fig. 1B) [45]. For successful tooth replacement regenerative therapy, a tooth developed from a bioengineered germ or a transplanted bioengineered mature tooth unit must be capable of being successfully grafted into the lost tooth region in an adult oral environment and achieve full functionality, including sufficient masticatory performance, biological cooperation with the periodontal tissues and afferent responsiveness to noxious stimulations in the maxillofacial region [1].
1) Transplantation of bioengineered tooth germ or a bioengineered mature tooth unit as a tooth replacement therapy

Successful tooth regenerative therapy, via transplantation of a bioengineered tooth germ into the lost tooth region, requires that the transplanted germ erupt and occlude properly with the opposing tooth in the adult jawbone. Tooth eruption is a cooperative regulatory mechanism that involves the tooth germ cell component and surrounding alveolar/jawbone [46]. Dental follicle cells migrate from near the surface of the enamel organs and dental papillae to form the cementum, periodontal ligament, and alveolar bone. These cells contribute to overlying bone resorption by enzymatic degeneration during growth and the axial movement of the teeth during the process of tooth eruption [47]. Bioengineered tooth germs can develop the correct structure in a tooth cavity, and normal tooth germs can develop and also erupt in a toothless diastema region [48]. Furthermore, in animal models, bioengineered tooth germs can form the correct tooth structure in the oral cavity [23] and can successfully erupt 37 days after transplantation. The bioengineered tooth subsequently reaches the occlusal plane and maintains occlusion with the opposing tooth from 49 days onwards [25••].

To produce a transplanted bioengineered tooth unit comprising a mature tooth, periodontal ligament and alveolar bone, the most critical consideration is whether the unit can be grafted into the tooth loss region with subsequent bone integration, which involves bone resorption of the alveolar bone of the bioengineered tooth unit through natural bone remodelling at the recipient site. Bioengineered teeth have been successfully grafted at 40 days and subsequently maintained with a functional periodontal ligament originating from the bioengineered tooth unit and traversing to the adjacent bone, implying successful bone integration [26•]. Furthermore, the enamel and dentin hardness of the bioengineered tooth components were in the normal range when analysed by the Knoop hardness test [25••, 26•]. These approaches demonstrate the potential to restore masticatory performance and natural tooth tissue through new technologies.

2) The biological response of a bioengineered tooth to mechanical stress

Tooth loss and functional disorders in periodontal ligaments cause fundamental problems for oral functions by affecting pronunciation, mastication, occlusion, and associated health issues [1]. The periodontal ligament plays an essential role in the pathogenic and physiological responses of teeth to extreme mechanical forces from bone remodelling accompanied by orthodontic tooth movement (Fig. 1B) [47]. Autologous tooth transplantation following a traumatic dental injury has demonstrated that teeth with healthy periodontal 
ligament cells can prevent ankylosis and attach successfully to the surrounding alveolar bone tissue [49]. By contrast, the absence of a periodontal ligament in osseo-integrated dental implants is associated with deficiencies of the essential tooth functions and in the natural structural relationship with the root and alveolar bone [7, 50]. Biological regenerative therapy using functional periodontal tissue has been shown to be effective in restoring normal tooth functions as an alternative to artificial therapy [51]. The periodontal ligaments of bioengineered teeth that erupted following transplantation of a bioengineered tooth germ resulted in the formation of mature tooth units with functional tooth movement equivalent to that of natural teeth. Bone remodelling was successfully reproduced via the proper localisation of osteoclasts and osteoblasts in response to mechanical stress. These findings indicate that a bioengineered tooth can replicate critical dental functions and subsequently lead to the restoration and re-establishment of functional teeth within the maxillofacial region $[25 \bullet \bullet, 26 \bullet]$.

\section{3) Proprioception potential of neuronal response in bioengineered teeth}

Teeth are a peripheral target organ for the sensory trigeminal and sympathetic nerves, both of which play important roles in homeostasis and protection [50]. The perceptive potential for noxious stimulation, including mechanical stress and pain, are important for proper tooth function. Trigeminal ganglional neurons, which innervate the pulp and PDL, can respond to these stimulations and transduce the perceptions to the central nervous system (Fig. 1B). It is anticipated that tooth regenerative therapies will be able to recover the neuronal perceptive ability of mechanical forces, which are lacking in implant patients [52]. Recently, nerve fibres, such as the sensory and sympathetic nerves, have been shown to innervate both the pulp and PDL of a regenerated tooth that had successfully erupted following the transplantation of not only bioengineered tooth germ, but also a bioengineered tooth unit $[25 \bullet \bullet, 26 \bullet$. These bioengineered teeth have the appropriate perceptive potential for noxious stimulations, such as pulp injury and orthodontic force, and can also properly transduce these stimulations to the central nervous system through c-Fos immunoreactive neurons [25••, 26•]. These findings indicate that bioengineered teeth can indeed restore the proprioceptive potential to identify and respond to noxious stimulation within the maxillofacial region.

\section{Future Perspectives for Tooth Regeneration}

There are several problems that must be solved before bioengineered teeth become feasible. To fully realise the practical clinical application of tooth regeneration, suitable cell sources must be identified. Ideally, tooth regenerative therapy should employ the patient's own cells to avoid immunological rejection [20]. Recent studies of stem cell biology and embryonic development have led to considerable advances in the potential of cell sources for tissue repair and organ regeneration, including tooth regenerative therapy $[10,18]$. Tooth tissue-derived stem cells such as DPSCs, SHED, SCAP and PDLSCs [13-16, 53] can differentiate into dental cell lineages and contribute to the turnover and supply of various cell populations. These lineages will be useful cell sources for stem cell transplantation therapy for dental tissue repair [12, 20], but there is no evidence that these stem cells have the potential to form whole teeth.

Current whole tooth regenerative therapy research is attempting to induce bioengineered tooth germ to develop a fully functioning tooth using embryonic tooth germ-derived epithelial and mesenchymal cells via the organ germ method $[23,24 \bullet, 25 \bullet \bullet, 26 \bullet]$. In the future, cell sources that have the tooth-forming ability to reproduce the epithelial and mesenchymal interactions for tooth organogenesis will need to be identified from the various tissue-derived stem cell populations isolated from patients. Other candidate cell sources for whole tooth regeneration include embryonic stem (ES) cells and induced pluripotent stem (iPS) cells, which are capable of differentiating into endoderm, ectoderm and mesoderm [54]. iPS cells have recently been generated from various oral tissues [55-57], but their application will require the establishment of reprogramming procedures for dental epithelium and mesenchyme fates. Although there are multiple candidates for tooth developmental genes that promote significant expression of dental epithelial and mesenchymal cells, the master genes responsible for tooth development remain to be discovered. An important issue for identifying a future tooth regenerative cell source is the identification of specific combinations of factors capable of reprogramming non-dental cells to dental epithelium and mesenchyme.

Tooth types such as incisors, canines, premolars and molars have unique morphological features that are programmed at predetermined sites in the oral cavity during tooth development. Several studies have proposed molecular mechanisms for tooth morphology regulation [30, 44•, 58]. Tooth size, crown and root shape are important considerations when generating a bioengineered regenerated tooth with proper functional occlusion and aesthetics.

\section{Conclusions}

The progress of regenerative technology is remarkable, and many patients and clinicians are anticipating the implementation of whole tooth regenerative therapy. Further studies are required to establish bioengineering technologies that can control tooth morphology, including tissue engineering using 
scaffolds, and the identification of morphogenesis-related genes and appropriate cytokines. Tooth regenerative therapy is now regarded as a crucial study model for future replacement regenerative therapies that can be applied to more complex organs, and will contribute substantially to the knowledge and technology required to regenerate organs $[20,21]$.

\section{Compliance with Ethics Guidelines}

Conflict of Interest Dr. Kei Nakajima, Dr. Masamitsu Oshima, and Dr. Takashi Tsuji each declare that there are no potential conflicts of interest relevant to this article.

Human and Animal Rights and Informed Consent This article does not contain any studies with human or animal subjects performed by any of the authors.

\section{References}

Papers of particular interest, published recently, have been highlighted as:

- Of importance

-. Of major importance

1. Proffit WR, Fields Jr HW, Sarver DM. Contemporary orthodontics. St. Louis: Mosby Press; 2004. p. 78-83.

2. Pispa J, Thesleff I. Mechanisms of ectodermal organogenesis. Dev Biol. 2003;262:195-205.

3. Tucker A, Sharpe P. The cutting-edge of mammalian development; how the embryo makes teeth. Nat Rev Genet. 2004;5:499-508.

4. Avery JK. Oral development and histology. New York: Thieme Press; 2002. p. 153-212.

5. Brenemark PI, Zarb GA. Tissue-integrated prostheses. In: Albrektsson T, editor. In osseointegration in clinical dentistry. Berlin: Quintessence Pub Co Press; 1985. p. 211-32.

6. Rosenstiel SF, Land MF, Fujimoto J. Contemporary fixed prosthodontics. St. Louis: Mosby Press; 2001. p. 209-430.

7. Huang GT, Sonoyama W, Liu Y, et al. The hidden treasure in apical papilla: the potential role in pulp/dentin regeneration and bioroot engineering. J Endod. 2008;34:645-51.

8. Brockes JP, Kumar A. Appendage regeneration in adult vertebrates and implications for regenerative medicine. Science. 2005;310:191923.

9. Atala A. Tissue engineering, stem cells and cloning: current concepts and changing trends. Expert Opin Biol Ther. 2005;5:87992.

10. Korbling M, Estrov Z. Adult stem cells for tissue repair - a new therapeutic concept? N Engl J Med. 2003;349:570-82.

11. Gurtner GC, Werner S, Barrandon Y, Longaker MT. Wound repair and regeneration. Nature. 2008;453:314-21.

12. Huang GT, Gronthos S, Shi S. Mesenchymal stem cells derived from dental tissues vs. those from other sources: their biology and role in regenerative medicine. J Dent Res. 2009;88:792-806.

13. Gronthos S, Mankani M, Brahim J, et al. Postnatal human dental pulp stem cells (DPSCs) in vitro and in vivo. Proc Natl Acad Sci USA. 2000;97:13625-30.

14. Miura M, Gronthos S, Zhao M, et al. SHED: stem cells from human exfoliated deciduous teeth. Proc Natl Acad Sci USA. 2003;100: 5807-12.
15. Sonoyama W, Liu Y, Yamaza T, et al. Characterization of the apical papilla and its residing stem cells from human immature permanent teeth: a pilot study. J Endod. 2008;34:166-71.

16. Seo BM, Miura M, Gronthos S, et al. Investigation of multipotent postnatal stem cells from human periodontal ligament. Lancet. 2004;364:149-55.

17. Thesleff I. Epithelial-mesenchymal signalling regulating tooth morphogenesis. J Cell Sci. 2003;116:1647-8.

18. Mantesso A, Sharpe P. Dental stem cells for tooth regeneration and repair. Expert Opin Biol Ther. 2009;9:1143-54.

19. Yen AH, Sharpe PT. Stem cells and tooth tissue engineering. Cell Tissue Res. 2008;331:359-72.

20. Ikeda E, Tsuji T. Growing bioengineered teeth from single cells: potential for dental regenerative medicine. Expert Opin Biol Ther. 2008;8:735-44.

21. Sharpe PT, Young CS. Test-tube teeth. Sci Am. 2005;293:34-41.

22. Duailibi SE, Duaibili MT, Vacanti JP, et al.: Prospects for tooth regeneration. Periodontol 2000 2006, 41:177-187.

23. Nakao K, Morita R, Saji Y, et al. The development of a bioengineered organ germ method. Nat Methods. 2007;4:227-30.

24. Oshima M, Ogawa M, Yasukawa M, Tsuji T. Odontogenesis methods in molecular biology volume 887. In: Kioussi C, editor. Generation of a bioengineered tooth by using a three-dimensional cell manipulation method (organ germ method). New York: Humana Press; 2012. p. 149-65. These protocols described tooth germ reconstitution by using the Organ Germ Method..

25.• Ikeda E, Morita R, Nakao K, et al. Fully functional bioengineered tooth replacement as an organ replacement therapy. Proc Natl Acad Sci USA. 2009;106:13475-80. This study demonstrated a therapeutic concept of fully functioning tooth regenerative therapy by the transplantation of a bioengineered tooth germ.

26. Oshima M, Mizuno M, Imamura A, et al. Functional tooth regeneration using a bioengineered tooth unit as a mature organ replacement regenerative therapy. PLoS One. 2011;6:e21531. This study indicated the potential for bioengineered mature tooth replacement as a future organ replacement regenerative therapy..

27. Jernvall J, Thesleff I. Reiterative signaling and patterning during mammalian tooth morphogenesis. Mech Dev. 2000;92:1929.

28. Zheng Y, Du X, Wang W, et al. Organogenesis from dissociated cells: generation of mature cycling hair follicles from skin-derived cells. J Invest Dermatol. 2005;124:867-76.

29. Toyoshima K, Asakawa K, Ishibashi N, et al. Fully functional hair follicle regeneration through the rearrangement of stem cells and their niches. Nat Commun. 2012;3:784.

30. Wei C, Larsen M, Hoffman MP, Yamada KM. Self-organization and branching morphogenesis of primary salivary epithelial cells. Tissue Eng. 2007;13:721-35.

31. Griffith LG, Naughton G. Tissue engineering-current challenges and expanding opportunities. Science. 2002;295:1009-14.

32. Caplan AI, Bruder SP. Mesenchymal stem cells: building blocks for molecular medicine in the 21st century. Trends Mol Med. 2001;7: 259-64.

33. Young CS, Terada S, Vacanti JP, et al. Tissue engineering of complex tooth structures on biodegradable polymer scaffolds. J Dent Res. 2002;81:695-700.

34. Duailibi MT, Duailibi SE, Young CS, et al. Bioengineered teeth from cultured rat tooth bud cells. J Dent Res. 2004;83: 523-8.

35. van Manen EH, Zhang W, Walboomers, et al. The influence of electrospun fibre scaffold orientation and nano-hydroxyapatite content on the development of tooth bud stem cells in vitro. Odontology. 2012. doi:10.1007/s10266-012-0087-9.

36. Sumita Y, Honda MJ, Ohara T, et al. Performance of collagen sponge as a 3-D scaffold for tooth-tissue engineering. Biomaterials. 2006;27: 3238-48. 
37. Honda MJ, Tsuchiya S, Sumita Y, et al. The sequential seeding of epithelial and mesenchymal cells for tissue-engineered tooth regeneration. Biomaterials. 2007;28:680-9.

38. Yen AH, Sharpe PT. Regeneration of teeth using stem cell-based tissue engineering. Expert Opin Biol Ther. 2006;6:9-16.

39. Zheng L, Yang F, Shen H, et al. The effect of composition of calcium phosphate composite scaffolds on the formation of tooth tissue from human dental pulp stem cells. Biomaterials. 2011;32: 7053-9.

40. Shackleton M, Vaillant F, Simpson KJ, et al. Generation of a functional mammary gland from a single stem cell. Nature. 2006;439:84-8.

41. Yamamoto H, Kim EJ, Cho SW, Jung HS. Analysis of tooth formation by reaggregated dental mesenchyme from mouse embryo. J Electron Microsc (Tokyo). 2003;52:559-66.

42. Hu B, Nadiri A, Kuchler-Bopp S, et al. Tissue engineering of tooth crown, root, and periodontium. Tissue Eng. 2006;12:2069-75.

43. Song Y, Zhang Z, Yu X, et al. Application of lentivirus-mediated RNAi in studying gene function in mammalian tooth development. Dev Dyn. 2006;235:1334-44.

44. Ishida K, Murofushi M, Nakao K, et al. The regulation of tooth morphologenesis is associated with epithelial cell proliferation and the expression of Sonic hedgehog through epithelial-mesenchymal interactions. Biochem Biophys Res Commun. 2011;405:455-61. This study showed that the crown width and cusp number of bioengineered tooth could be regulated by the cell-to-cell contact area..

45. Dawson PE. Functional occlusion: from TMJ to smile design. St. Louis: Mosby Press; 2006. p. 18-26.

46. Wise GE, Frazier-Bowers S, D'Souza RN. Cellular, molecular, and genetic determinants of tooth eruption. Crit Rev Oral Biol Med. 2002;13:323-34.
47. Wise GE, King GJ. Mechanisms of tooth eruption and orthodontic tooth movement. J Dent Res. 2008;87:414-34.

48. Ohazama A, Modino SA, Miletich I, Sharpe PT. Stem-cell-based tissue engineering of murine teeth. J Dent Res. 2004;83:518-22.

49. Tsukiboshi M. Autogenous tooth transplantation: a reevaluation. Int J Periodontics Restorative Dent. 1993;13:120-49.

50. Lindhe J, Lang NP, Karring T. Clinical periodontology and implant dentistry. New Jersey: Wiley-Blackwell Press; 2008. p. 99 107.

51. Bartold PM, Shi S, Gronthos S: Stem cells and periodontal regeneration. Periodontol 2000 2006, 40:64-172.

52. Hämmerle $\mathrm{CH}$, Wagner $\mathrm{D}$, Brägger $\mathrm{U}$, et al. Threshold of tactile sensitivity perceived with dental endosseous implants and natural teeth. Clin Oral Implants Res. 1995;6:83-90.

53. Sonoyama W, Liu Y, Fang D, et al. Mesenchymal stem cellmediated functional tooth regeneration in swine. PLoS One. 2006; 1:e79.

54. Takahashi K, Yamanaka S. Induction of pluripotent stem cells from mouse embryonic and adult fibroblast cultures by defined factors. Cell. 2006;126:663-76.

55. Egusa H, Okita K, Kayashima H, et al. Gingival fibroblasts as a promising source of induced pluripotent stem cells. PLoS one. 2010;5:e12743.

56. Yan X, Qin H, Qu C, et al. iPS cells reprogrammed from human mesenchymal-like stem/progenitor cells of dental tissue origin. Stem Cells Dev. 2010;19:469-80.

57. Miyoshi K, Tsuji D, Kudoh K, et al. Generation of human induced pluripotent stem cells from oral mucosa. J Biosci Bioeng. 2010;110: 345-50.

58. Cai J, Cho SW, Kim JY, et al. Patterning the size and number of tooth and its cusps. Dev Biol. 2007;304:499-507. 